\title{
Feasibility Study of Two-Dimensional Neutron-Resonance Thermometry using Molybdenum in 316 Stainless-Steel
}

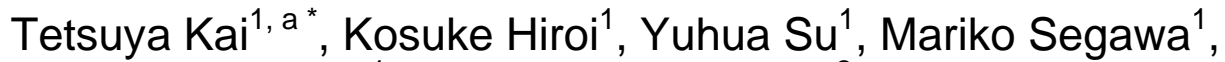 \\ Takenao Shinohara ${ }^{1}$, Yoshihiro Matsumoto ${ }^{2}$, Joseph D. Parker ${ }^{2}$, \\ Hirotoshi Hayashida ${ }^{2}$ and Kenichi Oikawa ${ }^{1}$ \\ ${ }^{1}$ Japan Atomic Energy Agency, Tokai-mura Naka-gun Ibaraki 319-1195, Japan \\ ${ }^{2}$ Comprehensive Research Organization for Science and Society, \\ Tokai-mura Naka-gun, Ibaraki 319-1106, Japan \\ a tetsuya.kai@j-parc.jp
}

Keywords: Temperature Measurement, RADEN, GEM Detector, Neutron Transmission, Reliability, Energy Dependent Imaging, Width, Time of Flight

\begin{abstract}
The energy-dependence of neutrons were measured through a 3-mm thick 316 stainless-steel with homogeneous temperatures from room temperature to about 500 degrees Celsius to investigate whether molybdenum contained in 316 stainless-steel was available as a sensor material for neutron resonance thermometry. Dips in the energy spectra around the 44.8 $\mathrm{eV}$ resonance of molybdenum were broadened with the increasing temperature, and a calibration line from width to temperature was obtained. A neutron measurement was also carried out for a 316 stainless-steel plate having a temperature distribution. By analyzing the width of the resonance at each position, a reasonable temperature distribution was obtained. Molybdenum contained in 316 stainless-steel was found to be useful for neutron resonance thermometry.
\end{abstract}

\section{Introduction}

A two-dimensional thermometry technique based on neutron resonance reactions derives the temperature of specified elements in an object by analyzing the Doppler broadening of a neutron resonance absorption width measured by a time-analyzing neutron imaging-detector. This technique is expected to be one of the important applications of the energy-resolved neutron imaging techniques. As a part of ongoing feasibility studies, the authors discussed the reliability of this technique using tantalum and tungsten from 26 to $285^{\circ} \mathrm{C}$ in a previous study [1]. Tantalum is the most commonly used sensor material for neutron resonance thermometry due to its suitable resonance properties (width, energy and magnitude), however, a more widely-available material has an advantage to promote applications of this technique.

The authors focused on molybdenum contained in 316 stainless-steel (with a weight fraction of 2-3 wt\%), which is a well-known and widely-used material in various fields. One molybdenum isotope, molybdenum-95 (natural abundance is 15.9\%), exhibits resonances of 15 kilo-barns at a neutron energy of $44.8 \mathrm{eV}$. The magnitude of this resonance cross section is large enough for resonance absorption imaging, and the thinner relative width of the resonance than that of the tantalum largest resonance at $4.3 \mathrm{eV}$ may bring about higher temperature sensitivity, while the resonance energy is about 10 times higher according to the evaluated nuclear data library, JENDL-4 [2]. This consideration of the resonance properties, and a calculation using the REFIT code $[3,4]$, indicated that molybdenum could be used for neutron resonance thermometry. Figure 1 shows neutron transmission rates of $50 \mu \mathrm{m}$ thick molybdenum around the resonance at temperatures between 25 and $500^{\circ} \mathrm{C}$ calculated by the REFIT code. The thickness of molybdenum is equivalent to the $3-\mathrm{mm}$ thick 316 stainless-steel containing $2.1 \%$ molybdenum. 
The broadening of the resonance shape with increasing temperature was simulated. It should be noted that the temperature effect might not be the same between pure molybdenum (used in the REFIT calculation) and molybdenum contained within an alloy since the Doppler broadening caused by the increase of thermal vibrations of the molybdenum atoms can be affected by the crystallographic structure of the composite material. Thus, the authors were motivated to measure the broadening of the molybdenum resonance in 316 stainless-steel to confirm the availability of such temperature measurement method. A demonstration measurement of the temperature distribution of a 316 stainless-steel plate was carried out.

\section{Experimental}

Calibration measurement. The measurements in this study were performed at the energy-resolved neutron imaging system, RADEN [5], installed at JPARC. For the measurement, the proton beam power was $400 \mathrm{~kW}(25 \mathrm{~Hz})$ with a single-bunch operation mode. Thermal and cold neutrons were eliminated from the incident beam by a 1-mm thick cadmium filter, and the neutron beam size was collimated to $50 \times 50 \mathrm{~mm}^{2}$ using stainless-steel and boron-impregnated polyethylene blocks. The broadening of the resonance of molybdenum in 316 stainless-steel was measured by using a $3 \mathrm{~mm}$ thick plate at temperatures of 23, 99, 146, 194, 244, 293, 343, 393, 444 and $494^{\circ} \mathrm{C}$. The stainless-steel plate was set inside a vacuum quartz tube (inner diameter: ca. $36 \mathrm{~mm}$ ) of a furnace [1] having an Inconel heater surrounded by a gold-coated reflector, and the temperatures of the plate were measured by a thermocouple attached on the plate. A copper plate (1 mm in thickness) was attached to the stainlesssteel plate to ensure a homogeneous temperature distribution. The energy-dependent transmitted neutron intensity was imaged using a gas-electron multiplier (GEM) detector [6] located at a neutron flight path of $23.8 \mathrm{~m}$. The sample area within the neutron beam was $30 \times 50 \mathrm{~mm}^{2}$.

Neutron intensities through the sample at 23 and $494^{\circ} \mathrm{C}$ are shown in Fig. 2 as a function of neutron energy. The statistical uncertainties at the resonance energy (bottoms of dips) were 0.3 and $0.4 \%$ for the 23 and $494^{\circ} \mathrm{C}$, respectively. It was clearly recognized that dips corresponding to the $44.8 \mathrm{eV}$

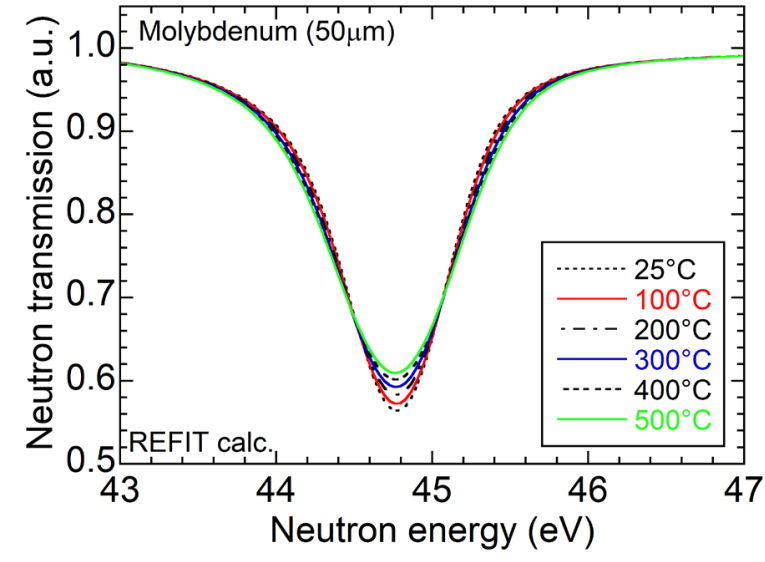

Fig. 1 Neutron transmission rates of $50 \mu \mathrm{m}$ thick molybdenum calculated with the REFIT code at various temperatures.

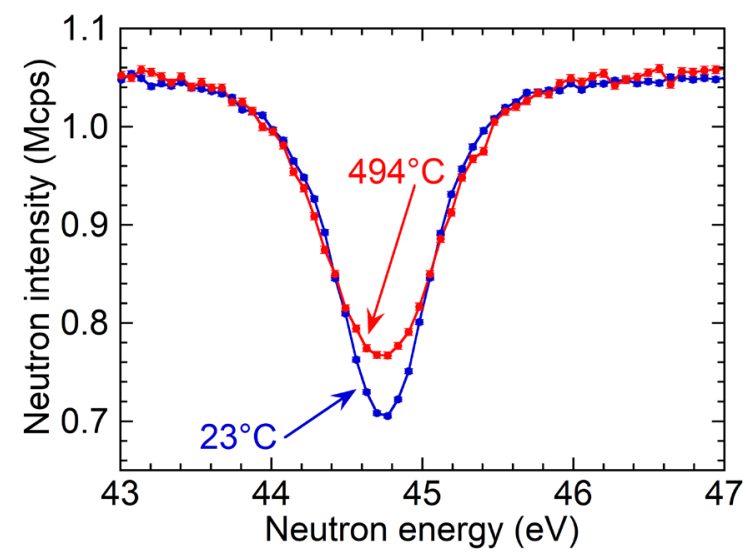

Fig. 2 Neutron intensities around the 44.8 eV molybdenum resonance through a $3 \mathrm{~mm}$ thick 316 stainless steel at 23 and $494^{\circ} \mathrm{C}$.

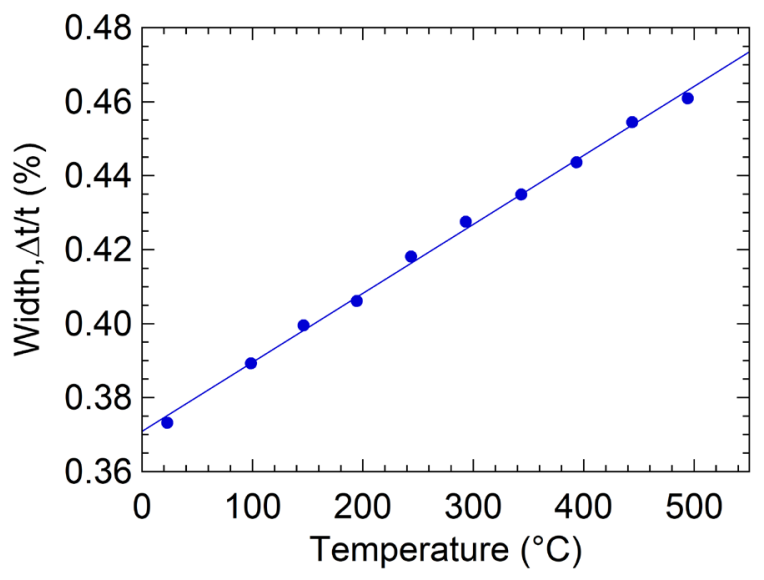

Fig. 3 Measured widths of molybdenum resonance in a $3 \mathrm{~mm}$ thick 316 stainlesssteel at various temperatures. The solid line is a linear fit result. 
molybdenum resonance were broadened at the higher temperature. To estimate the broadening effect, the widths of the resonance relative to the temperature were obtained by fitting the measured spectra assuming a Gaussian-shaped resonance cross section as shown in Fig. 3 . The fitted Gaussian width was adopted as the measured width. A calibration line to derive temperature from the width of the resonance was obtained by fitting these results. A reasonable linearity was recognized between temperature and width although fitting errors (not shown in Fig. 3) were rather large due to using the simple Gaussian function without taking into account pulse shapes of incident neutron beam.

Demonstration measurement. A stainless-steel plate of 3-mm thickness was prepared to demonstrate a measurement of the two-dimensional distribution of temperature. The same material as the calibration measurement was used. A schematic view of the plate is shown in Fig. 4 (a). A slit of $1 \mathrm{~mm}$ in width was made from the top to the center of the viewed area of $50 \times 50$ $\mathrm{mm}^{2}$ expecting a temperature gap. An aluminum nitride heater was attached at the right upper part of the plate, and was kept at $300^{\circ} \mathrm{C}$. The lower part of the plate was used to secure it on a thermal insulation board. The temperatures of the lower part were monitored by two thermocouples. Infrared thermography was also used to monitor the temperature distribution. The plate was coated with black heat-resistant paint to ensure high temperature emissivity of infrared rays. Figure 4 (b) shows an example of the temperature distribution obtained by the infrared thermography. A temperature distribution caused by the heat transfer from the heater and an isolation by the slit was observed. The temperature increased in 20 minutes after turning on the heater, and then became stable. Under these stable temperature conditions, the transmitted neutrons were measured by the GEM detector located at the same position as the calibration measurement. The distance from the sample plate to the detector was $275 \mathrm{~mm}$. The GEM detector produced neutron spectra in the field-of-view of $100 \times 100 \mathrm{~mm}^{2}$ divided by $128 \times 128$ pixels. As shown in a neutron radiograph at $44.8 \mathrm{eV}$ in Fig. 4 (c), neutrons were collimated to 67 $\times 67$ pixels $\left(52 \times 52 \mathrm{~mm}^{2}\right)$, and the slit in the plate was confirmed to be in the field-of-view. The cadmium filter was used as in the calibration measurement. The L/D was 400. The neutron measurements were carried out for both heater ON and OFF conditions.

The proton beam power was $500 \mathrm{~kW}$ with a double-bunch operation mode, in which two proton bunches were contained in a pulse injected to the neutron source at $25 \mathrm{~Hz}$. The second bunch was delayed by $0.6 \mu$ s relative to the first one, and the width of each bunch was $0.1 \mu$ s.
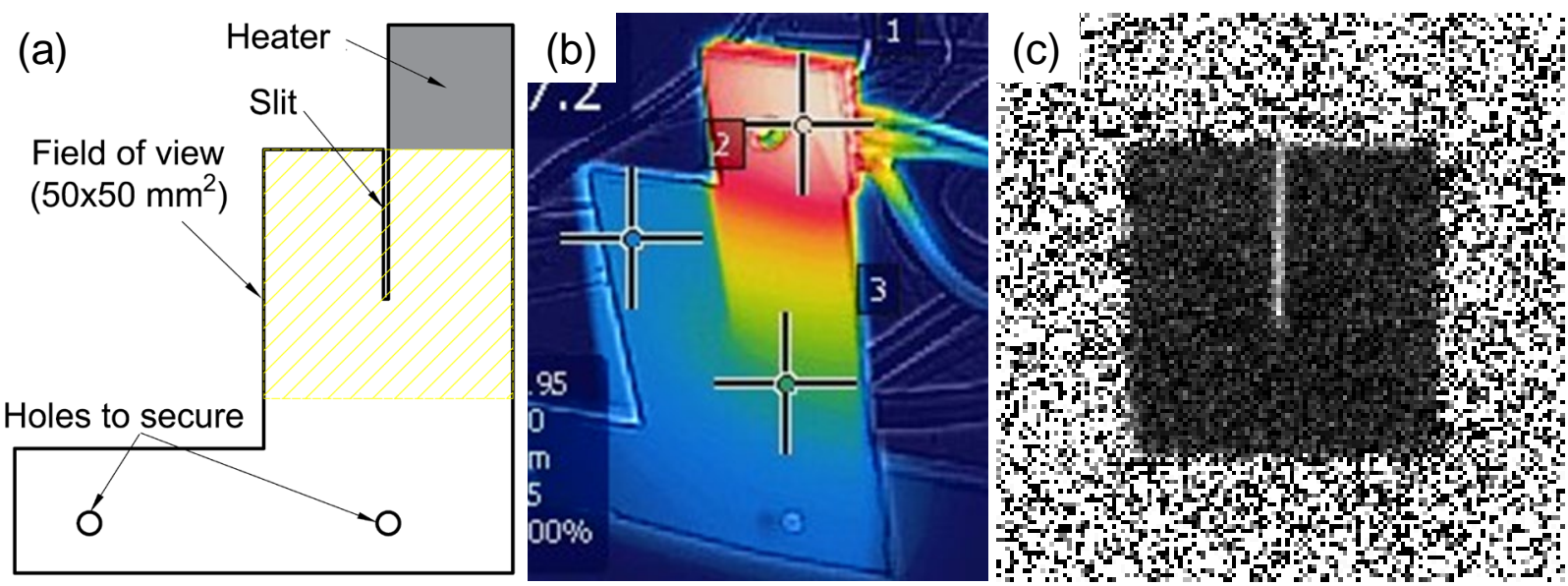

Fig. 4 (a) Schematic view of sample plate made by 316 stainless-steel of 3-mm thickness, (b) temperature distribution during heating measured by infrared thermography. The sample plate was coated with black heat-resistant paint to ensure high emissivity, (c) neutron radiograph at $44.8 \mathrm{eV}$. 
The neutron spectrum measured in the heater OFF condition was used to correct the difference of the operation mode. The normalized neutron intensities around the $44.8 \mathrm{eV}$ resonance at room temperature measured in the single-bunch (calibration measurement) and double-bunch (demonstration) modes are compared in Fig. 5. The width at $22^{\circ} \mathrm{C}$ for the double-bunch mode was $0.021 \%(0.05 \mu \mathrm{s})$ larger than that expected by the calibration line obtained in single-bunch mode. Although the difference of operation mode effected somewhat the width of the measured resonance dip, it was not as large as the temporal difference of the bunches. The calibration line for the single-bunch mode was modified to increase width by $0.021 \%$ in order to obtain the calibration line for the double-bunch mode as shown in Fig. 6. This discrepancy was accidentally caused in upgrading accelerator power from $400 \mathrm{~kW}$ to $500 \mathrm{~kW}$.

\section{Results and discussions}

The measured neutron intensity spectra in $20 \times 20$ pixels $\left(15.6 \times 15.6 \mathrm{~mm}^{2}\right)$ were averaged by scanning the position in one-pixel steps to improve the statistic accuracy. The averaged neutron spectra in a $48 \times 48$ matrix were obtained from the $67 \times 67$ neutron spectra. The resonance dips in the spectra were fitted in the same manner as in the calibration measurement, and the two-dimensional distribution of widths was produced. Figure 7 shows the distribution of temperature obtained by converting the width distribution at a heater temperature of $300^{\circ} \mathrm{C}$ by applying the calibration line in Fig. 6. The temperature was highest near the heater, and decreased with increasing distance from the heater to the bottom. The effect of the slit was also shown at the left side of the figure, where the temperature rapidly decreased at the slit. The derived temperature at the upper right corner near the heater was about $210-220^{\circ} \mathrm{C}$, while that measured by the infrared thermography was about $230^{\circ} \mathrm{C}$. The derived temperature at the upper left corner, the lowest temperature area, was $20-40^{\circ} \mathrm{C}$, while about $50^{\circ} \mathrm{C}$ was indicated by the infrared thermography. The temperature difference seemed to be larger for the lower temperature, although a more detailed, quantitative comparison was difficult because the averaging area was rather large compared to the gradient of the temperature. The temperature distribution derived by analyzing the neutron

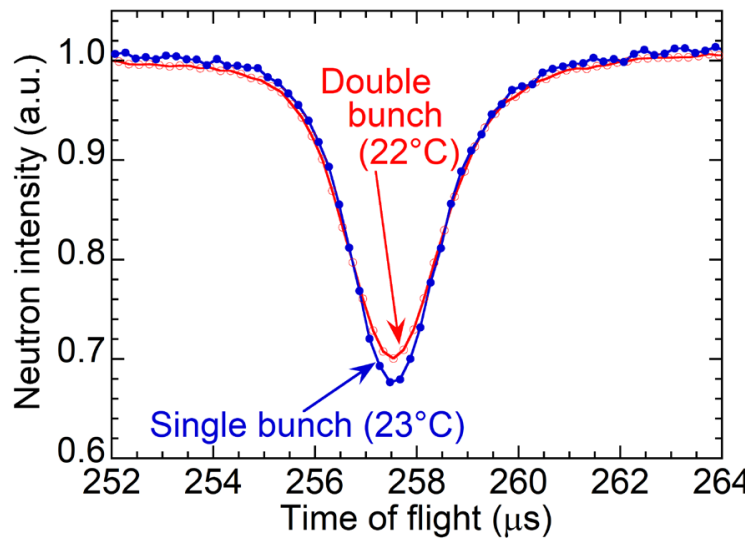

Fig. 5 Normalized neutron intensities around molybdenum resonance measured in proton beam operation modes of single and double bunch through room temperature samples.

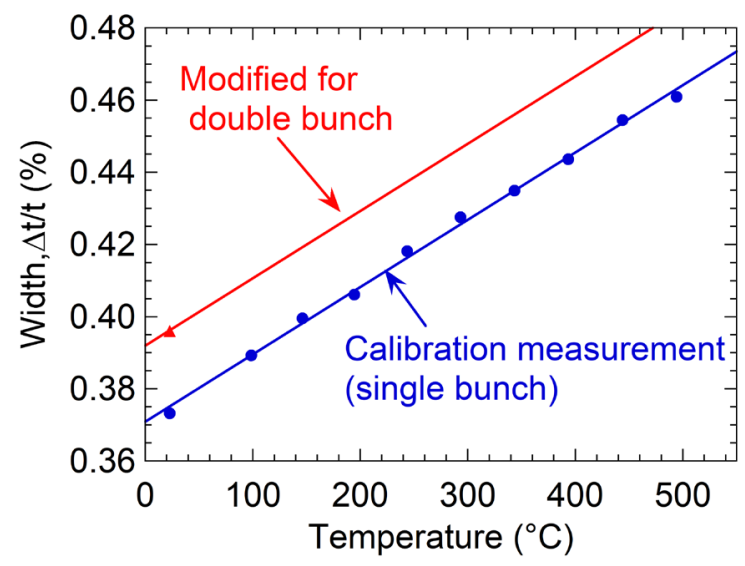

Fig. 6 Calibration lines obtained in the single-bunch mode and modified for the double-bunch mode.

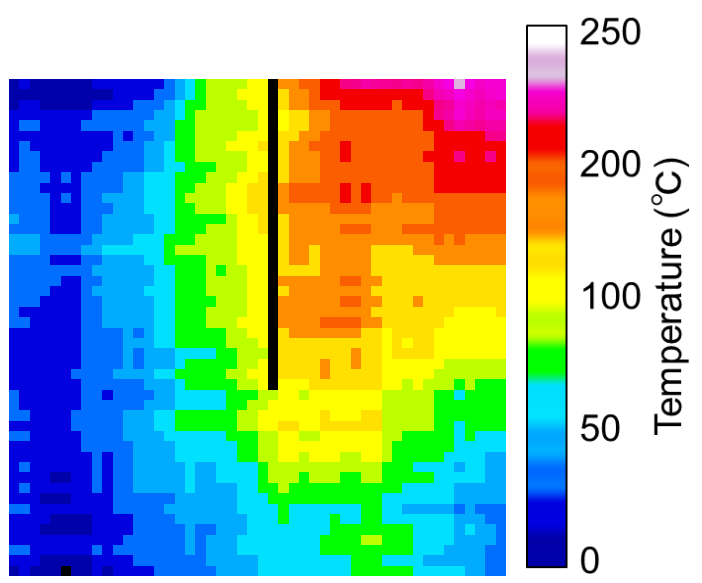

Fig. 7 Two-dimensional distribution of temperature of 316 stainless-steel obtained by analyzing molybdenum $44.8 \mathrm{eV}$ resonance. Black line indicates the position of slit. 
resonance, however, qualitatively reproduced the temperature distribution of the stainless-steel well.

\section{Summary}

The energy-dependent neutron spectra were measured through a 316 stainless-steel plate of 3mm thickness at homogeneous temperatures between 23 and $494^{\circ} \mathrm{C}$. The Doppler broadening of the $44.8 \mathrm{eV}$ resonance of molybdenum in 316 stainless-steel was observed, and the widths of the resonance were obtained as a function of temperature by fitting a Gaussian-shaped resonance cross section. It was found that the width increased linearly with the temperature in this range, and the calibration line was obtained. This calibration measurement was carried out in the singlebunch mode of the proton accelerator, while the demonstration measurement was performed in the double-bunch mode. The effect of the different operation modes was corrected by the result of the demonstration measurement at room temperature to produce a modified calibration line.

The demonstration measurement was carried out using a 3-mm thick stainless-steel plate with a heater (set at $300^{\circ} \mathrm{C}$ ) attached at the upper corner, just outside the field-of-view. Although some difference was observed between the temperatures derived by analyzing the neutron resonance spectra and those measured by the infrared thermography, the temperature distribution was qualitatively well reproduced considering the rather large averaging area of $15.6 \times 15.6 \mathrm{~mm}^{2}$ for the neutron resonance measurement. Based on this result, it was concluded that molybdenum in 316 stainless-steel could be used as a sensor material for the neutron resonance thermometry technique. A Gaussian-shaped resonance cross section was assumed for simplicity in this study, however, a function taking into account pulse shapes of neutron beam, intrinsic shape of resonance cross section, background, etc. would have great advantages in reproducing neutron transmission spectra. Such sophisticated function will be utilized to conduct detailed reliability estimation and to promote practical applications.

\section{Acknowledgement}

These measurements were carried out under the "Instrument Group Use” proposal (Proposal No.: 2018I0022) of the MLF J-PARC.

\section{References}

[1] T. Kai, K. Hiroi, Y. Su, T. Shinohara, J. D. Parker, et al., Reliability estimation of neutron resonance thermometry using tantalum and tungsten, Phys. Procedia 88 (2017) 306-313. https://doi.org/10.1016/j.phpro.2017.06.042

[2] K. Shibata, O. Iwamoto, T. Nakagawa, N. Iwamoto, A. Ichihara, S.et al., JENDL-4.0: A New Library for Nuclear Science and Engineering, J. Nucl. Sci. Technol. 48 (2011) 1-30.

[3] M.C. Moxon, T. C. Ware, C. J. Dean, Users’ guide for REFIT-2009-10, UKNSF(2010)P243, UK Nuclear Science Forum, 2010.

[4] H. Hasemi, M. Harada, et al., Evaluation of nuclide density by neutron resonance transmission at the NOBORU instrument in J-PARC/MLF, Nucl. Instrum. Method. A 773 (2014) 137-149. https://doi.org/10.1016/j.nima.2014.11.036

[5] T. Shinohara, T. Kai, K. Oikawa, M. Segawa, et al., Final design of the energy-resolved neutron imaging system "RADEN" at J-PARC, J. Phys.: Conf. Series 746 (1) (2016) 012007. https://doi.org/10.1088/1742-6596/746/1/012007

[6] S. Uno, T. Uchida, M. Sekimoto, T. Murakami, K. Miyama, M. Shoji, et al., Twodimensional neutron detector with GEM and its applications. Physics Procedia 26 (2012) 142. https://doi.org/10.1016/j.phpro.2012.03.019 